\title{
Hypothalamic dysfunction revealed by magnetic resonance diffusion tensor imaging in childhood leukemia survivors treated with cranial radiotherapy but not in craniopharyngioma survivors
}

Cecilia Follin, Sigridur Fjalldal, Daniel Svärd, Danielle van Westen, Sanaz Gabery, Åsa Petersen, Jimmy Lätt, Lars Rylander, Eva Marie Erfurth

\section{Conclusion}

\section{4 years after CRT and chemotherapy, DTI detects hypothalamic dysfunction in ALL survivors}

The survivors with overweight were presented with worse hypothalamic damage compared to survivors with normal weight. While CP without HT tumour involvement survivors seemed to be unaffected, the present data suggests important hypothalamic dysfunction after CRT in ALL survivors

\section{Background}

- Metabolic complications, including obesity, are frequent in acute lymphoblastic leukemia (ALL) survivors treated with cranial radiotherapy (CRT) in childhood

- Such complications are potentially mediated by the hypothalamus (HT), a regulator of energy expenditure

- Childhood onset (CO) Craniopharyngioma (CP) survivors without HT involvement are often spared gross obesity problems

- Magnetic resonance diffusion tensor imaging (DTI) provides information on the tissue microstructure of the brain as quantified by its parameters fractional anisotrophy (FA), mean diffusivity (MD), axial diffusivity (AD), radial diffusivity (RD)

\section{Aim}

Since MD in the HT is reportedly increased in obese compared to non-obese subjects, we investigated DTI in the HT in these two patient populations to find out HT's involvement in the obesity in ALL survivors

\section{Participants}

Delineation of the hypothalamus

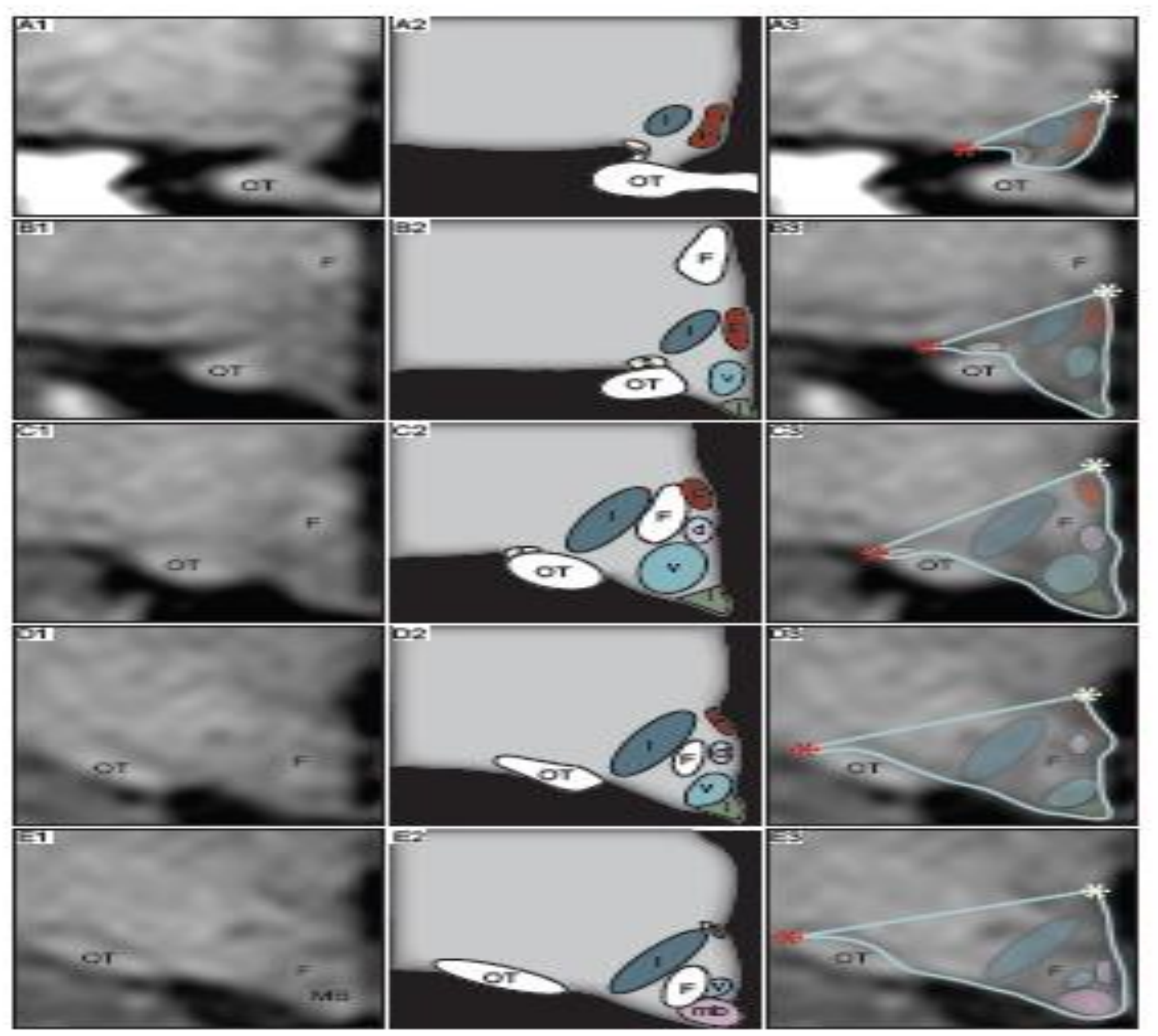

- 27 ALL survivors (median age 34 years (27-46 years), treated with a CRT dose of 24 Gy and on complete hormone supplementation, were investigated 34 years after diagnosis

- 17 CO-CP survivors (36 years (18-49 years) with complete hormone supplementation but without HT involvement according to peroperative neurosurgical assessment

- Comparisons were made to 27 age and sex matched controls regarding DTI parameters in the HT as well as for BMI, fat mass, fat free mass and waist/hip measurements

Results

DTI in hypothalamus (HT) in ALL survivors $(n=29)$ vs $\mathrm{CP}$ survivors $(\mathrm{n}=17)$ and control subjects $(\mathrm{n}=27)$

DTI in hypothalamus in 29 ALL survivors and in $17 \mathrm{CO}$ CP survivors compared to 27 healthy matched controls

\begin{tabular}{|c|c|c|c|c|c|}
\hline & \begin{tabular}{|l} 
ALL survivors \\
$(\mathrm{n}=29)$ \\
Median (range)
\end{tabular} & \begin{tabular}{|l|} 
CP survivors (n=17) \\
Median (range)
\end{tabular} & \begin{tabular}{|l} 
Controls $(\mathbf{n}=27)$ \\
Median (range)
\end{tabular} & \begin{tabular}{|l} 
PALL vs \\
Controls
\end{tabular} & \begin{tabular}{|l|l} 
PCP vs \\
Controls
\end{tabular} \\
\hline \multicolumn{6}{|l|}{ 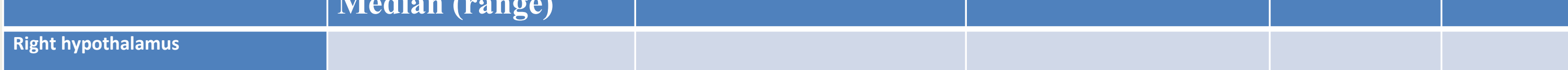 } \\
\hline tional anisotrophy & $0.27(0.20-0.34)$ & $0.29(0.19-0.35)$ & $0.29(0.25-0.35)$ & 0.04 & NS \\
\hline Mean diffusisity & $1.13(0.89-1.24)$ & $0.97(0.85-1.10)$ & $1.00(0.77-1.21)$ & $<0.001$ & NS \\
\hline \begin{tabular}{|l|l} 
Axial dififusivity \\
\end{tabular} & $1.41(1.18-1.64)$ & $1.21(1.11-1.37)$ & $1.25(1.02-1.49)$ & $<0.001$ & NS \\
\hline Radiali Diffuswivity & $0.99(0.74-1.19)$ & $0,85(0.72-0.99)$ & $0.86(0.65-1.07)$ & $<0.001$ & NS \\
\hline \multicolumn{6}{|l|}{ Lef hyothalalmus } \\
\hline Fractional anisotrophy & $0.30(0.20-0.35)$ & $0.32(0.22-0.36)$ & $0.31(0.25-0.34)$ & NS & NS \\
\hline Mean diffusisvity & $\begin{array}{l}.0 .00(0.76-0.05) \\
1.00\end{array}$ & $0.87(0.72-1.01)$ & $0.93(0.78-1.12)$ & 0.01 & NS \\
\hline Axial diffusivity & $1.27(0.97-1.37)$ & $1.14(0.97-1.31)$ & $1.18(1.01-1.39)$ & 0.01 & NS \\
\hline Radiala Diffusuvity & $0.87(0.66-0.97)$ & $0.73(0.60-0.89)$ & $0.79(0.65-0.98)$ & 0.01 & NS \\
\hline
\end{tabular}

DTI in hypothalamus in ALL survivors with BMI $\geq 25$ compared to control subjects with $B M I \geq 25$
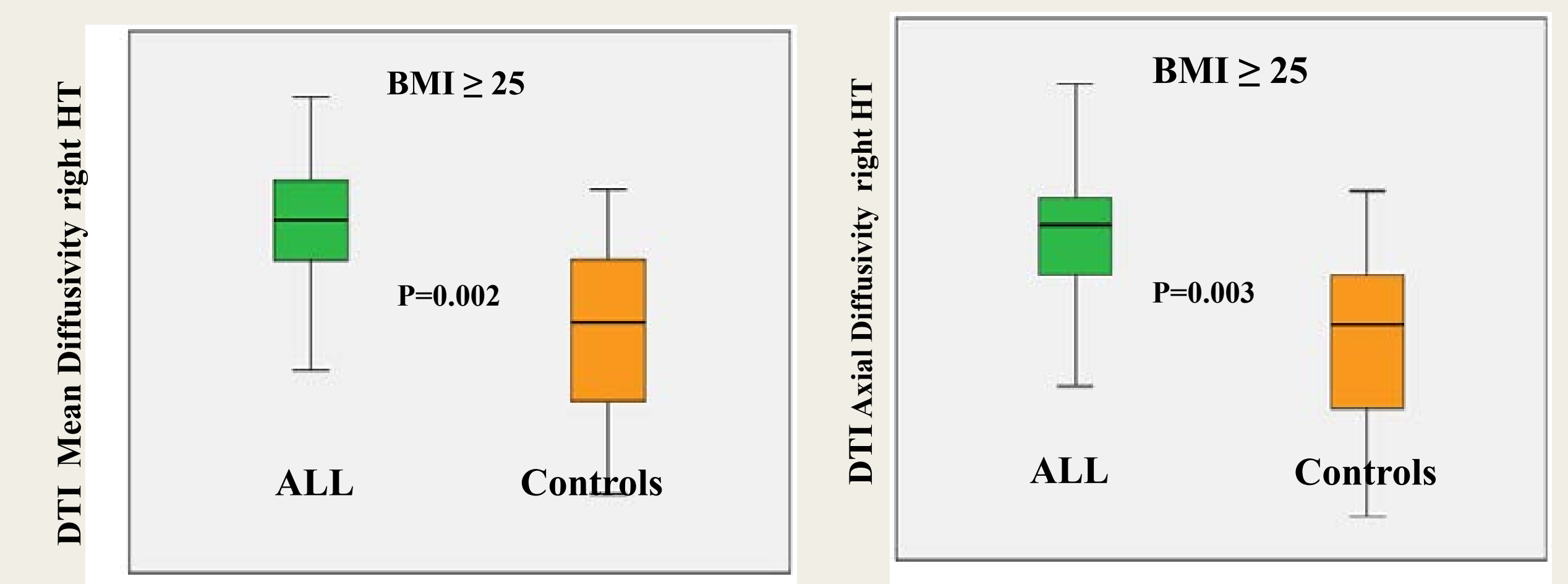
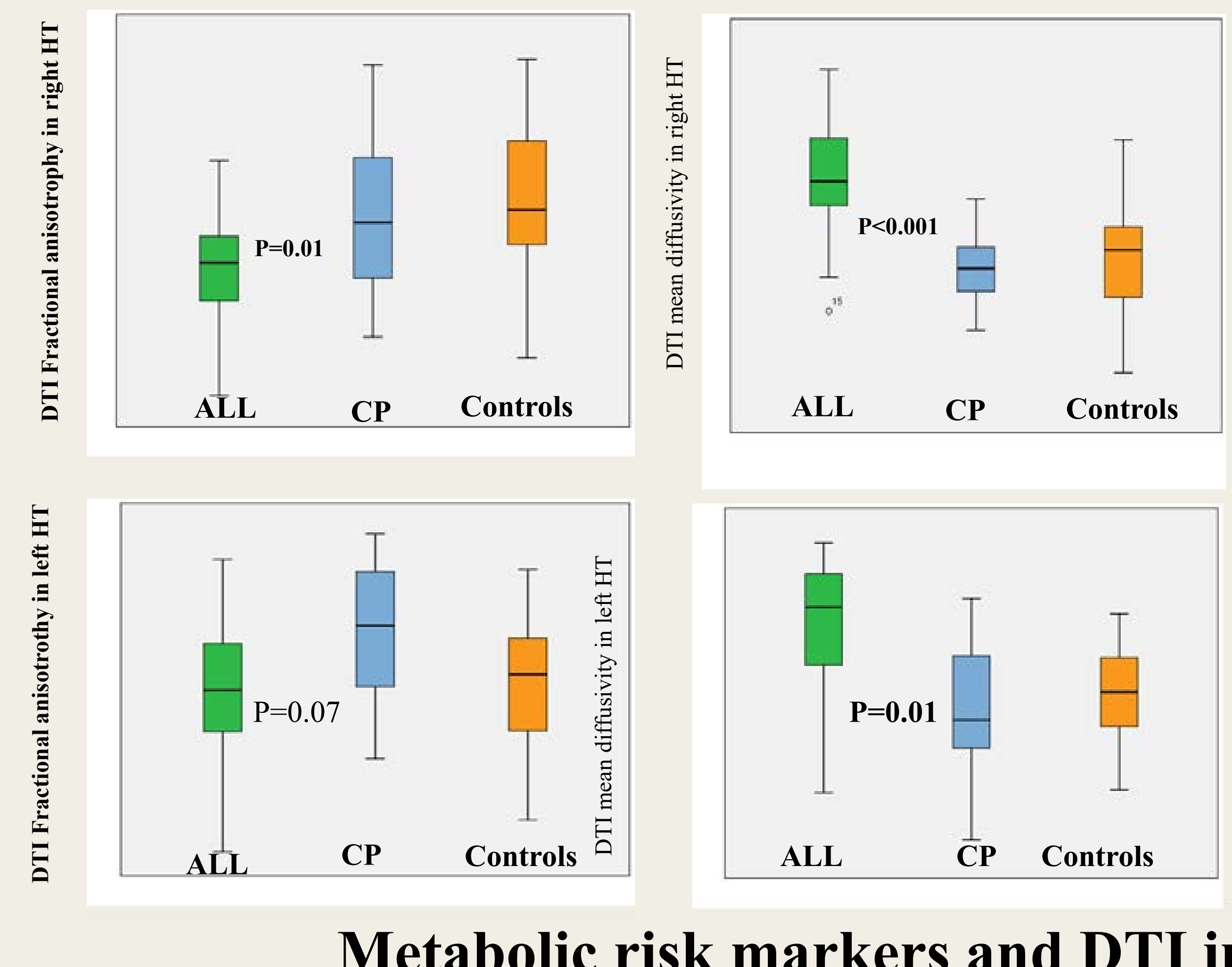

No treatment is currently available for these hypothalamic problems The mainstay is tailored healthy life-style support and proper hormone replacement

Metabolic risk markers and DTI in hypothalamus in 19 ALL survivors with $B M I \geq 25$ compared to ALL survivors with $\mathrm{BMI}<25$

\begin{tabular}{|c|c|c|c|}
\hline & \begin{tabular}{|l|} 
ALL survivors BMI $\geq 25$ \\
$\mathrm{~N}=19$
\end{tabular} & $\begin{array}{l}\text { ALL survivors BMI }<25 \\
N=9\end{array}$ & $\mathbf{P}$ \\
\hline Age at diagnosis & $4.4(1.1-17)$ & $3.2(1.1-13)$ & NS \\
\hline Height $(\mathrm{cm})$ & $1.61(1.47-1.85)$ & $1.73(1.46-1.85)$ & \\
\hline Weight (kg) & $73.9(55.9-123.5)$ & $64,2(38.7-74.8)$ & 0.009 \\
\hline BMI $\left(\mathrm{kg} / \mathrm{m}^{2}\right)$ & $29(25.2-39.1)$ & $23.2(18.2-24.9)$ & $<0.001$ \\
\hline Fatfree mass (kg) & $41.2(30.3-73)$ & $45.8(26-52.6)$ & NS \\
\hline Fat mass (kg) & $30.2(14-55.3)$ & $23.4(10.6-30)$ & 0.007 \\
\hline Glucose (mmolLL) & $5.3(4.1-13.6)$ & $5.2(4.9-6.5)$ & NS \\
\hline Insulin (mIE/L) & $11(2-32)$ & $11(4-26)$ & NS \\
\hline Leptin $(\mu \mathrm{g} / \mathrm{L})$ & $29.5(5.0-90.0)$ & $13(2.2-23)$ & 0.01 \\
\hline Ghrelin (ng/L) & $1145(556-2400)$ & $1140(634-3670)$ & NS \\
\hline \multicolumn{4}{|l|}{ Right hypothalamus } \\
\hline Mean diffusivity & $1.17(0.97-1.34)$ & $1.08(0.89-1.77)$ & 0.03 \\
\hline Axial diffusivity & $1.44(1.21-1.69)$ & $1.37(1.18-1.57)$ & 0.02 \\
\hline
\end{tabular}

